\title{
Nutritional status of adolescents in selected government and private secondary schools of Addis Ababa, Ethiopia
}

\author{
Yoseph Gebreyohannes ${ }^{1}$, Solomon Shiferaw ${ }^{2}$, Balem Demtsu ${ }^{3,}$,, Gessessew Bugssa ${ }^{4}$ \\ ${ }^{1}$ Ethiopian Health and Nutrition Research Institute, Addis Ababa, Ethiopia \\ ${ }^{2}$ Department Public health, Medical faculty, Addis Ababa University, Addis Ababa, Ethiopia \\ ${ }^{3}$ Department of Midwifery, college of Health Sciences, Mekelle University, Mekelle, Ethiopia \\ ${ }^{4}$ Institute of Biomedical Sciences, College of Health Sciences, Mekelle University, Mekelle, Ethiopia \\ Email address: \\ yosephgy@gmail.com (Y. Gebreyohannes), bugssag@gmail.com (G. Bugssa), balemdim@gmail.com (B. Demtsu)
}

\section{To cite this article:}

Yoseph Gebreyohannes, Solomon Shiferaw, Balem Demtsu, Gessessew Bugssa. Nutritional Status of Adolescents in Selected Government and Private Secondary Schools of Addis Ababa, Ethiopia. International Journal of Nutrition and Food Sciences.

Vol. 3, No. 6, 2014, pp. 504-514. doi: 10.11648/j.ijnfs.20140306.13

\begin{abstract}
Background: Changes in the nutritional intake combined with increasingly sedentary life styles and increasing urbanization has led to the emergence of chronic disease as a major new health threat. Objective: To assess and compare nutritional status of adolescents and analyze the risk factors associated to/with overweight/obesity in government and private secondary schools of Addis Ababa, 2012. Methods: A comparative cross sectional study comprising 1024 adolescents of government and private secondary schools of Addis Ababa was conducted from February 02, 2012 to June $28,2012$. Information on socio-demographic data, eating habits and physical activity was collected using interviews. Measurements on weight and height were made using standardized weighing scales and measuring boards, respectively. Height-for-age and body mass index-for-age were compared to the 2007 WHO growth reference. Data were entered using Epi info version 3.5.1, WHO AnthroPlus and analyzed using SPSS version 16. Results: Overall, the prevalence of stunting (low height-for-age), underweight (low body mass index-for-age) and overweight/obese (high body mass index-for-age) in all school adolescents was 7.2\% (95\% CI; 5.8, 9.0), 6.2\% (95\% CI; 4.9, 8.0) and 8.5\% (95\% CI; 6.9, 10.4), respectively. Adolescents in government schools were significantly more likely to be undernourished [stunting; 51(10.0\%) versus 23(4.5\%) and underweight; 36(7.0\%) versus $28(5.5 \%)$ ] compared to their counterparts in private schools (P-value $<0.05)$. Adolescents in private schools were overweight/obese compared to those in government schools [65(12.7\%) versus 22(4.3\%); OR=3.2 (95\% CI; 1.9, 5.3)]. Conclusions and recommendations: The findings of this investigation showed that being in a private school, lacking daily breakfast and consumption of animal products more than once a day are significantly associated with overweight/Obese during adolescence. Results of this study emphasize the need for educational interventions at early ages involving the whole family to promote optimal nutritional status.
\end{abstract}

Keywords: Comparative Cross Sectional, Nutritional Status, Adolescents, Addis Ababa, Ethiopia

\section{Introduction}

Adolescence is a decisive period of development because it represents the transition between life as a child, and life as an adult covering the ages between 10-19 years. During this crucial period, dietary patterns have vital impact on lifetime nutritional status and health [1, 2]. Increased nutritional needs at this juncture relate to the fact that adolescents gain up to $50 \%$ of their adult weight, more than $20 \%$ of their adult height, and $50 \%$ of their adult skeletal mass during this period. However, adolescents face a series of serious nutritional challenges which would affect this rapid growth spurt as well as their health as adults [3].

The main nutritional problems affecting adolescent populations worldwide include under nutrition (in terms of stunting and wasting), overweight and obesity which are emerging public health problems during this time [3-5]. Worldwide, $10 \%$ of adolescents are overweight with obesity prevalence varying between $2-3 \%$. The global averages reflect prevalence that varies from $10 \%$ in Africa and Asia to more than $20 \%$ in the USA and Europe $[6,7]$. The nutrition and epidemiologic transition has its own impact on changes 
in diet and activity patterns, leading to the development of a double burden of malnutrition. The changes in the nutritional intake combined with increasingly sedentary life styles resulting from food market, globalization and increasing urbanization has led to the emergence of chronic disease as a major new health threat $[6,8,9]$. Adolescent obesity is associated with serious medical problems, including high blood pressure, adverse lipoprotein profiles, diabetes mellitus, atherosclerotic cerebrovascular disease, coronary heart disease, colorectal cancer, and death from all causes as well as lower educational attainment and higher rates of poverty $[10,11]$.

Adolescence is a period of transition between childhood and adulthood and also a significant period of human growth and maturation. Poor nutritional status during adolescence is an important determinant of health outcomes. Short stature in adolescents resulting from chronic under nutrition is associated with reduced lean body mass and deficiencies in muscular strength and working capacity [12]. Adolescents receive very little attention; as a result, there is limited understanding of the problem of dual burden and how best to manage it. The newly developed 2007 World Health Organization (WHO) growth reference for children and adolescents, however, simplify the problem and is used by this time for the assessment of thinness (low BMI(body mass index)-for-age), overweight and obesity (high BMI-for-age) and stunting (low height-for-age)[6, 13].

The increase in the prevalence of obesity in children and adolescents is a reality in all developed or developing countries associated with serious medical problems and lower educational attainment. It also dramatically increases the risk of adult obesity which is alarming because obesity is credited with 400,000 deaths annually in the United States [10]. A cross sectional study conducted on prevalence of childhood and adolescent overweight and obesity among elementary schools in Addis Ababa indicated that prevalence of underweight (BMI for age less than $5^{\text {th }}$ percentile), overweight (BMI for age $85^{\text {th }}$ percentile and above) and obesity (BMI for age $95^{\text {th }}$ percentile and above) were $13 \%$, $7.6 \%$ and $0.9 \%$, respectively [14]. A study conducted on nutritional status of adolescent girls from rural communities of Tigray, northern Ethiopia, showed that the prevalence of stunting and thinness were $26.5 \%$ and $58.3 \%$, respectively [3]. Another study conducted in Ambo town showed that underweight was prevalent in males than females $(29.8 \%$ versus $24.6 \%$ ) with total prevalence of $27.5 \%$ whereas overweight was more prevalent in females than in males (3.8\%\% versus $1.7 \%$ ) with overall prevalence of $4.3 \%$ [2].

The WHO and FAO (Food and Agriculture Organization) expert group found convincing evidence that high intake of energy dense foods is a risk factor for obesity. It also found that heavy marketing of fast foods and high intakes of sugar sweetened drinks were probable risk factors and that large portion sizes was a possible risk factor for obesity [10]. Changes in patterns of physical activity and the adoption of more sedentary lifestyles are likely to be important factors behind obesity. A study showed that only $31 \%$ of US adults report that they engage in regular leisure-time physical activity (defined as either three sessions per week of vigorous physical activity lasting 20 minutes or more or five sessions per week of light-to-moderate physical activity lasting 30 minutes or more). About $35 \%$ of high school students report that they participate in at least 60 minutes of physical activity on 5 or more days of the week, and only $30 \%$ of students report that they attend physical education class daily. As children get older, participation in regular physical activity decreases dramatically [16]. An Independent Television Commission survey conducted on England showed that 4-15 years old children watch Television (Tv) approximately 2.5 hours of a day. The research also showed that a strong correlation between the number of hours spent watching TV and increased risk of obesity [15].

In general, there is no documented study done on adolescent nutritional status in Addis Ababa. Therefore, examination of the two extremes of malnutrition may provide insight into the nature of dual burden and how to direct prevention strategies, both for the immediate problem of underweight, and the rapidly increasing problem of overweight/obesity in adolescents. This study, is therefore, aimed at assessing the level of adolescent nutritional status and identifying factors contributing to adolescent overweight and obesity in the selected government and private secondary schools of Addis Ababa.

\section{Materials and Methods}

\subsection{Study Design, Area and Period}

A School based cross-sectional comparative study was conducted among randomly selected government and private secondary school adolescents of Addis Ababa from February 02, 2012 to June 28, 2012.

Sample size: The sample size was calculated using twosample proportion formula, P1(14\%) the prevalence of overweight/obesity among adolescents in government high schools from a study conducted elsewhere [22, 25], and P2(24\%) prevalence of overweight/obesity among adolescents in private high schools (a 10\% difference was assumed to signify public health significance for lack of previous studies). Taking Pooled population, $\mathrm{P}=19 \%, \mathrm{Z} \alpha /{ }_{2}=$ 1.96 at $95 \%$ level of confidence, $Z_{\beta}=0.84$ for $80 \%$ power of the test, $r=1$, Proportion of group 1 to group 2 (Government to Private) was taken as equal $1: 1, \mathrm{n} 1$ was calculated to be 240 and $n_{2}=240$, thus $n$ was 480 . Adding 5\% non-response rate gives the total sample size of 504 . Considering design effect, 2(504 x 2) the sample size was calculated to be 1008 . Based on these assumptions, the required sample size was 1008 but later it was adjusted to 1024(512 from government and 512 from private secondary school students) to get equal number of students from each section (cluster).

Sampling technique: Multi stage sampling method was used to select the study participants. Out of the 39 high schools constituting 13 government and 26 private schools, 12(4 government and 8 private schools) were randomly 
selected. Secondly, using lottery method 64 clusters (32 clusters from each school type) were identified considering the available resource and different age distribution. From each of the selected 12 schools all sections of grade 9, 10, 11 and 12 were separately enlisted, two sections from each grade in government and one section from each grade in private schools were randomly selected using lottery method to identify 64 clusters ( 32 clusters from each school type). Out of each selected section (cluster), 16 eligible subjects were randomly selected using systematic random sampling. Those who had obvious physical deformity and were pregnant during data collection were excluded from the study.

Quality control measures: A standardized questionnaire was used, containing the variables except for physical activity which was assessed using the standard questionnaire "International Physical Activity Questionnaire (IPAQ)". The food consumption was evaluated using the standard foods frequency questionnaire for adolescents classified as: $\left(f_{1}\right)$ never; $\left(f_{2}\right)$ less than once a month; $\left(f_{3}\right)$ one to three times per month; $\left(f_{4}\right)$ once a week; $\left(f_{5}\right)$ two to four times a week; $\left(f_{6}\right)$ once per day and $\left(f_{7}\right)$ more than twice per day. Body weight was measured using a standard digital balance with a precision of $0.1 \mathrm{Kg}$. The students wore only light clothes during measurement of body weight. The scales were carefully handled and calibrated every morning by placing standard calibration weights of $2 \mathrm{Kg}$ iron bars on the scale to ascertain accuracy. Besides, height was measured using a measuring board with a precision of $0.1 \mathrm{~cm}$.

Data analysis: Data was entered using Epi info version 3.5.1. The WHO 2007 growth reference was used as a standard reference for classifying nutritional status of adolescents using WHO Anthroplus software. SPSS software version 16 was used for statistical analysis. Descriptive statistics and Logistic regression analysis were used as appropriate. Statistical significance was set at $\mathrm{p}<0.05$.

\subsection{Study Variables}

Dependent Variables: Overweight/obesity, Underweight, Stunting

Independent variables: Sex, Age, Educational status of the family, Marital Status, Religion, Economic status, Eating habit, Physical activity, Sedentary lifestyle.

Ethical Considerations: Ethical clearance and approval was obtained from the Research Ethics Committee at the School of Public Health, Addis Ababa University. Further permission was obtained from each school. The students were informed about the objective of the study and then data was collected after oral consent was obtained from each student.

\section{Results}

\subsection{Socio-Demographic Characteristics}

A total of 1024 students participated in the study giving a response rate of $100 \%$. As displayed in Table 1 , out of the 1024 respondents, 512(50.0\%) were from government schools, $521(50.9 \%)$ males, $606(59.2 \%)$ belonged to the age group 17-19 years and $1011(98.7 \%)$ were single. Majority $(67.0 \%)$ of the respondents were Christian Orthodox followed by Muslim (19.6\%). Four hundred ninety four (48.2\%) fathers and 343(33.5\%) mothers of the students were reported to have completed grade12, respectively. Average family size of the students household was $6(\mathrm{SD}=2.2)$. Regarding occupational status, 262(25.6\%), 223(21.8\%) and $328(32 \%)$ of fathers' occupation and 149(14.6\%), 160(15.6\%) and $246(24 \%)$ of mothers' occupation were private employees, civil servants, and merchants, respectively.

Table 1. Socio-demographic characteristics of adolescents among government and private secondary schools of Addis Ababa, 2012.

\begin{tabular}{|c|c|c|c|c|}
\hline \multirow{3}{*}{ Variables } & \multicolumn{2}{|l|}{ School type } & \multirow{2}{*}{ Total } & \multirow{3}{*}{ P-Value } \\
\hline & Government & Private & & \\
\hline & $n(\%)$ & $n(\%)$ & $\mathbf{N}(\%)$ & \\
\hline \multicolumn{5}{|l|}{ Sex } \\
\hline Male & $248(48.4)$ & $273(53.3)$ & $521(50.9)$ & \multirow[t]{2}{*}{0.12} \\
\hline Female & $264(51.6)$ & $239(46.7)$ & $503(49.1)$ & \\
\hline \multicolumn{5}{|l|}{ Age group } \\
\hline $13-16$ & $175(34.2)$ & $243(47.5)$ & $418(40.8)$ & \multirow[t]{2}{*}{$<0.01$} \\
\hline $17-19$ & $337(65.8)$ & $269(52.5)$ & $606(59.2)$ & \\
\hline \multicolumn{5}{|l|}{ Religion } \\
\hline Orthodox & $332(64.8)$ & $354(69.1)$ & $686(67)$ & \multirow{4}{*}{$<0.05$} \\
\hline Protestant & $53(10.4)$ & $58(11.3)$ & $111(10.8)$ & \\
\hline Muslim & $119(23.2)$ & $82(16.0)$ & 201(19.6) & \\
\hline Others & $8(1.6)$ & $18(3.6)$ & $26(2.6)$ & \\
\hline \multicolumn{5}{|l|}{ Marital Status } \\
\hline Single & $508(99.2)$ & $503(98.2)$ & 1011(98.7) & \multirow{2}{*}{0.54} \\
\hline Divorced/ widowed & $1(0.2)$ & $2(0.4)$ & $3(0.3)$ & \\
\hline
\end{tabular}

\subsection{Nutritional Status of Adolescents}

As indicated in Table 2, the mean age, height, and weight were 16.8 years, $162.8 \mathrm{~cm}$, and $53.2 \mathrm{~kg}$, respectively. BAZ (BMI-for-Age Z-score) and HAZ (Height-for-Age Z-score) of the adolescents were -0.48 , and -0.72 , respectively. Males were taller and heavier in both government and private schools on average by $10 \mathrm{cms}$ and $4 \mathrm{Kgs}$, respectively. Mean BAZ and HAZ were significantly higher in private schools than government schools with mean difference of $0.24(95 \%$ 
CI; $0.11,0.36)$ and $0.36(95 \% \mathrm{CI} ; 0.25,0.46)$ respectively with $\mathrm{P}<0.01$. Mean $\mathrm{BAZ}$ was also significantly higher in females than males with mean difference of 0.49 (95\% CI; $0.37,0.62$ ) with $\mathrm{P}<0.01$.

Table 2. Mean age, height, weight, BAZ and HAZ of adolescents by sex among government and private secondary schools of Addis Ababa, 2012.

\begin{tabular}{|c|c|c|c|c|c|c|c|c|c|}
\hline \multirow{2}{*}{ Characteristics (Mean) } & \multicolumn{3}{|c|}{ Government Schools $(n=512)$} & \multicolumn{3}{|c|}{ Private schools $(n=512)$} & \multicolumn{3}{|c|}{ Over all $(n=1024)$} \\
\hline & Male & Female & Total & Male & Female & Total & Male & Female & Total \\
\hline Age (years) & 16.9 & 17.1 & 16.9 & 16.6 & 16.6 & 16.6 & 16.8 & 16.9 & 16.8 \\
\hline Height (cm) & 166.4 & 156.9 & 161.5 & 169.4 & 158.2 & 164.2 & 167.9 & 157.6 & 162.8 \\
\hline Weight (kg) & 53.0 & 50.2 & 51.6 & 57.1 & 52.3 & 54.8 & 55.1 & 51.2 & 53.2 \\
\hline BAZ & -0.91 & -0.31 & -0.6 & -0.55 & -0.14 & -0.36 & -0.72 & -0.23 & -0.48 \\
\hline HAZ & -0.94 & -.87 & -0.9 & -0.48 & -0.62 & -0.54 & -0.7 & -0.75 & -0.72 \\
\hline
\end{tabular}

The mean BMI-for-age and height-for-age Z-scores were compared to the 2007 WHO growth reference population (Figure 1, Figure 2). Both Z-scores of the study population were below the mean scores of the WHO growth reference population. The overall prevalence of stunting (low heightfor-age), underweight (low BMI-for-age) and overweight/obese (high BMI-for-age) in all school adolescents was 7.2\% (95\% CI; 5.8, 9.0), 6.2\% (95\% CI; 4.9, $8.0)$ and $8.5 \%(95 \% \mathrm{CI} ; 6.9,10.4)$ respectively (Figure 3$)$.

Comparison of nutritional status based on school type showed that more adolescents are underweight and stunted in government schools than private schools $(7.0 \%$ versus $5.5 \%$;
P-value 0.30$)$ and $(10 \%$ versus $4.5 \%$; with $\mathrm{P}<0.01)$ respectively.

Conversely, more students in private schools are significantly overweight/obese compared to those in government schools $(12.7 \%$ versus $4.3 \%$; with $\mathrm{P}<0.01)$ (Figure 3). Comparison of nutritional status of adolescents based on sex is indicated in Figure 4. Accordingly, males are significantly underweight than females $(9.8 \%$ versus $2.6 \%$; with $\mathrm{P}<0.01)$. On the other hand, more females were considerably obese compared to males $(11.3 \%$ versus $5.8 \%$; with $\mathrm{P}<0.05)$.

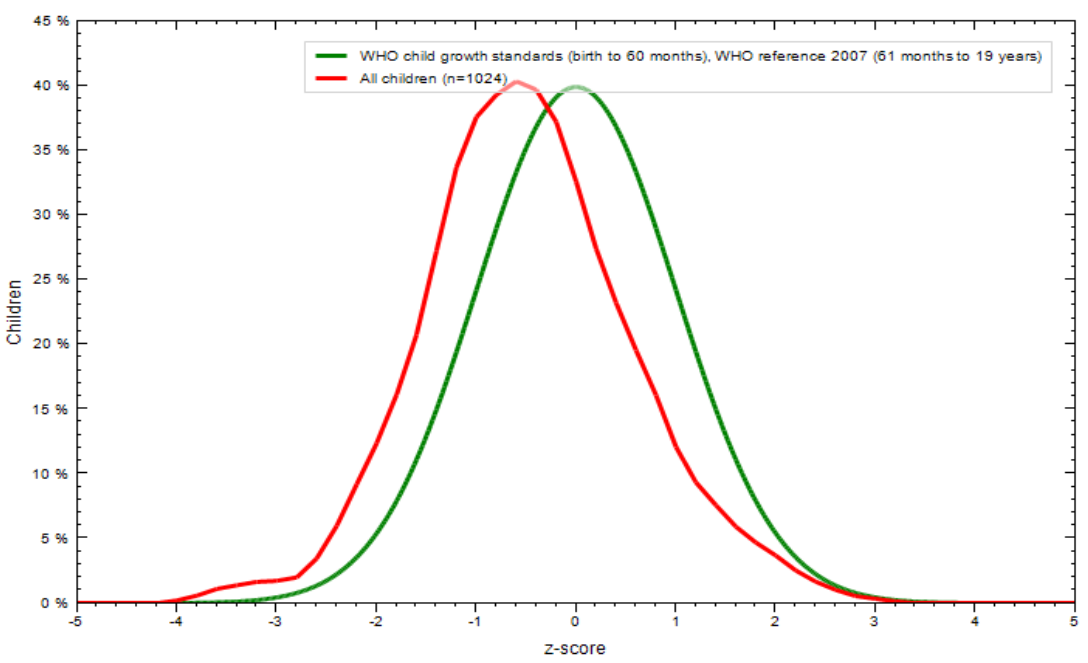

Figure 1. Comparison of BMI-for-age (BAZ) of the study population with the 2007 WHO growth reference populations $(n=1024)$.

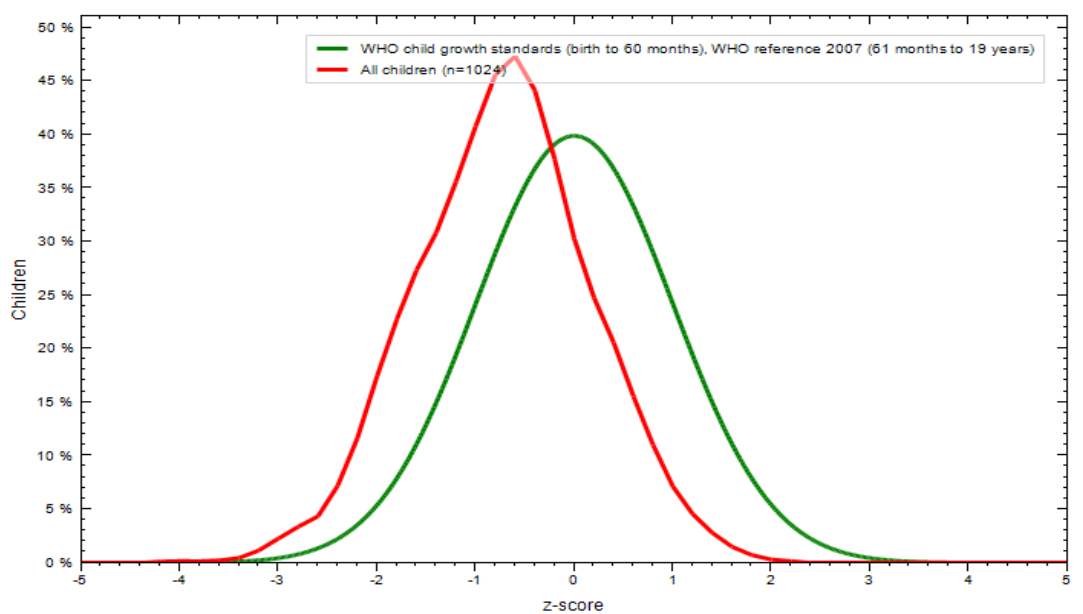

Figure 2. Comparison of Height-for-age (HAZ) of the study population with the 2007 WHO reference populations (n=1024). 


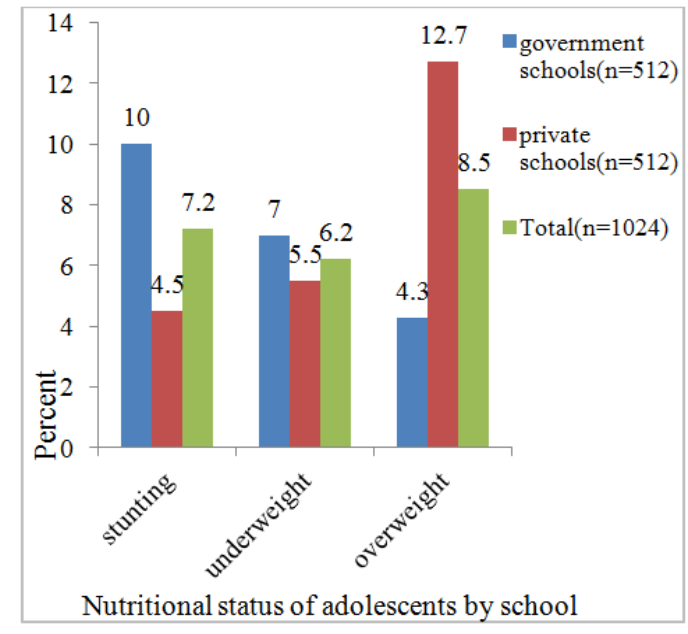

Figure 3. Nutritional status of adolescents among government and private secondary schools, Addis Ababa, 2012(n=1024).

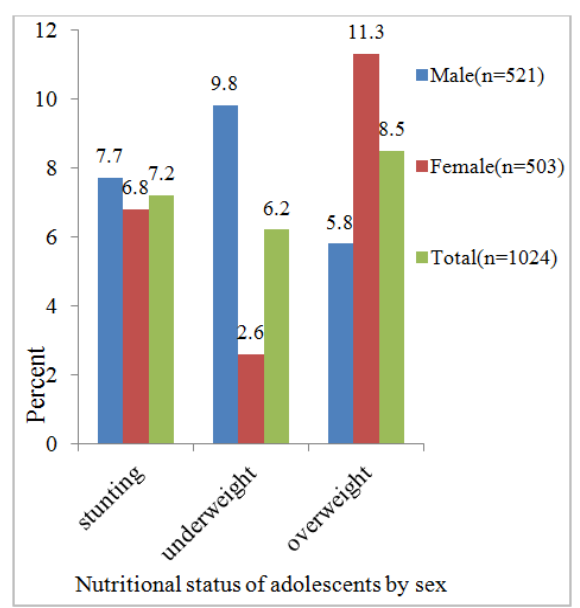

Figure 4. Nutritional status of adolescents by sex in government and private secondary schools of Addis Ababa, 2012(n=1024).

The mean BAZ for females were found to be comparable to $2007 \mathrm{WHO}$ growth reference while the mean BAZ for males were below the mean scores of the WHO growth reference population. The mean HAZ of both sexes was comparable but below the mean Height-for-age Z-score of the 2007 WHO growth reference population.

\subsection{Meal Pattern and Physical Activity}

As indicated in Table 3, consumption patterns of adolescents for various foods from eight different food groups were studied with the help of Food Frequency Questionnaire (FFQ). Of the study subjects, 51(5\%) took milk and milk products more than once a day, 78(7.6\%) took meat, egg and fish more than once a day.

Eating Habit: Frequency of meal pattern of participants was three meals per day i.e. breakfast, lunch and dinner which were typical meal patterns. From the total sampled participants, 766(74.8\%) took breakfast daily, 239(23.3\%) took breakfast sometimes and 19(1.9\%) never took breakfast at all. The majority, $858(83.3 \%)$ took lunch daily which was the main meal of the day and $140(13.7 \%)$ took lunch sometimes while $26(2.5 \%)$ never took lunch. Seven hundred seventy one $(75.3 \%)$ took dinner regularly all days of the week, 219(21.4\%) took dinner sometimes and 34(3.3\%) never took dinner. School recess and snack was taken daily by $465(45.4 \%)$ and $553(54 \%)$ of the respondents.

Sedentary life style of the participants showed that 483(47.2\%) spent more than two hours per day on TV, Video games and Computer; $23.9 \%, 51.6 \%$, and $24.5 \%$ of the participants slept $<6 \mathrm{hrs}, 6-9 \mathrm{hrs}$ and $>9 \mathrm{hrs}$ per day, respectively.

Concerning physical activity, adolescents were evaluated using International Physical Activity Questionnaire (IPAQ). Data collected with IPAQ questions were analyzed as categorical variables. The categories are 'low' (no activity is reported or some activity is reported but not enough to meet Categories 'moderate' or 'high'), 'moderate' (5 or more days of any combination of walking, moderate-intensity or vigorous intensity activities achieving a minimum of at least 600 minutes per week) and 'high' (7 or more days of any combination of walking, moderate- or vigorous-intensity activities accumulating at least 3000 minutes per week). Accordingly $354(34.6 \%), 660(64.5 \%)$ and $10(1 \%)$ were categorized as having low, moderate and high physical activity respectively, with males being more active $(\mathrm{P}<0.01)$ (Table 4).

Table 3. Meal pattern according to Food Frequency Questionnaire (FFQ) of adolescents among government and private secondary schools of Addis Ababa, 2012.

\begin{tabular}{|c|c|c|c|c|c|}
\hline \multirow{2}{*}{ Variables } & \multirow{2}{*}{ Level } & \multicolumn{2}{|l|}{ School type } & \multirow{2}{*}{ Total(n=1024) Number $(\%)$} & \multirow{2}{*}{ P-value } \\
\hline & & Government $(\mathrm{n}=512) \mathrm{n}(\%)$ & Private(n=512) n(\%) & & \\
\hline \multirow{3}{*}{ Cereal \& grains } & Two to four times per week & $8(1.6)$ & $17(3.3)$ & $25(2.4)$ & \multirow{3}{*}{$<0.01$} \\
\hline & Once a day & 102(19.9) & $63(12.3)$ & $165(16.1)$ & \\
\hline & Twice or more per day & $402(78.5)$ & $432(84.4)$ & $834(81.5)$ & \\
\hline \multirow{5}{*}{ Vegetables } & Never & $23(4.5)$ & $21(4.1)$ & $44(4.3)$ & \multirow{5}{*}{$<0.01$} \\
\hline & Less than once per month & $52(10.2)$ & $17(3.3)$ & $69(6.7)$ & \\
\hline & One to three times per month & $72(14.1)$ & $38(7.4)$ & $110(10.7)$ & \\
\hline & Once a week & $133(26.0)$ & $113(22.1)$ & $246(24.0)$ & \\
\hline & Two to four times per week & $162(31.6)$ & $190(37.1)$ & $352(34.4)$ & \\
\hline
\end{tabular}




\begin{tabular}{|c|c|c|c|c|c|}
\hline \multirow{2}{*}{ Variables } & \multirow{2}{*}{ Level } & \multicolumn{2}{|l|}{ School type } & \multirow{2}{*}{ Total(n=1024) Number (\%) } & \multirow{2}{*}{ P-value } \\
\hline & & Government (n=512) n(\%) & Private(n=512) n(\%) & & \\
\hline & Once a day & $51(10.0)$ & $96(18.8)$ & $147(14.4)$ & \\
\hline & Twice or more per day & $19(3.7)$ & $37(7.2)$ & $56(5.5)$ & \\
\hline & Never & $15(2.9)$ & $6(1.2)$ & $21(2.1)$ & \\
\hline & Less than once per month & $78(15.2)$ & $17(3.3)$ & $95(9.3)$ & \\
\hline & One to three times per month & $76(14.8)$ & $46(9.0)$ & $122(11.9)$ & \\
\hline \multirow[t]{7}{*}{ Fruits } & Once a week & $143(27.9)$ & $103(20.1)$ & $246(24.0)$ & $<0.01$ \\
\hline & Two to four times per week & $125(24.4)$ & $167(32.6)$ & $292(28.5)$ & \\
\hline & Once a day & $57(11.1)$ & $126(24.6)$ & $183(17.9)$ & \\
\hline & Twice or more per day & $18(3.5)$ & $47(9.2)$ & $65(6.3)$ & \\
\hline & Never & $80(15.6)$ & $43(8.4)$ & $123(12.0)$ & \\
\hline & Less than once per month & $97(18.9)$ & $37(7.2)$ & $134(13.1)$ & \\
\hline & One to three times per month & $110(21.5)$ & $72(14.1)$ & $182(17.2)$ & \\
\hline \multirow[t]{7}{*}{ Milk, Yogurt \& cheese } & Once a week & $109(21.3)$ & $88(17.2)$ & 197(19.2) & $<0.01$ \\
\hline & Two to four times per week & $62(12.1)$ & $105((20.5)$ & $167(16.3)$ & \\
\hline & Once a day & $37(7.2)$ & $133(26.0)$ & $170(16.6)$ & \\
\hline & Twice or more per day & $17(3.3)$ & $34(6.6)$ & $51(5.0)$ & \\
\hline & Never & $54(10.5)$ & $6(1.2)$ & $60(5.9)$ & \\
\hline & Less than once per month & $146(28.5)$ & $35(6.8)$ & $181(17.7)$ & \\
\hline & One to three times per month & $119(23.2)$ & $65(12.7)$ & $184(18.0)$ & \\
\hline \multirow[t]{7}{*}{ Meat, egg \& fish } & Once a week & $105(20.5)$ & $99(19.3)$ & 204(19.9) & $<0.01$ \\
\hline & Two to four times per week & $60(11.7)$ & $180(35.2)$ & $240(23.4)$ & \\
\hline & Once a day & $16(3.1)$ & 61(11.9) & $77(7.5)$ & \\
\hline & Twice or more per day & $12(2.3)$ & $66(12.9)$ & $78(7.6)$ & \\
\hline & Never & $350(68.4)$ & $385(75.2)$ & $735(71.8)$ & \\
\hline & Less than once per month & $89(17.4)$ & $62(12.1)$ & $151(14.8)$ & \\
\hline & One to three times per month & $30(5.8)$ & $26(5.1)$ & $56(5.5)$ & \\
\hline \multirow[t]{7}{*}{ Beans } & Once per week & $22(4.3)$ & $18(3.5)$ & $40(3.9)$ & $<0.05$ \\
\hline & Two to four times per week & $12(2.3)$ & $10(2.0)$ & $22(2.1)$ & \\
\hline & Once a day & $9(1.8)$ & $8(1.6)$ & $17(1.7)$ & \\
\hline & Twice or more per day & - & $3(0.5)$ & $3(0.2)$ & \\
\hline & Never & $60(11.7)$ & $3(0.6)$ & $63(6.2)$ & \\
\hline & Less than once per month & $135(26.4)$ & $45(8.8)$ & $180(17.6)$ & \\
\hline & One to three times per month & $79(15.4)$ & $54(10.5)$ & $133(13.0)$ & \\
\hline \multirow[t]{7}{*}{ Sweet $\&$ sugars } & Once a week & $76(14.8)$ & $99(19.3)$ & $175(17.1)$ & $<0.01$ \\
\hline & Two to four times per week & $76(14.8)$ & $111(21.7)$ & $187(18.3)$ & \\
\hline & Once a day & $60(11.7)$ & $113(22.1)$ & $173(16.9)$ & \\
\hline & Twice or more per day & $26(5.1)$ & $87(17.0)$ & $113(11.0)$ & \\
\hline & Never & $309(60.4)$ & $337(65.8)$ & $646(63.1)$ & \\
\hline & Less than once per month & $107(20.9)$ & $65(12.7)$ & $172(16.8)$ & \\
\hline & One to three times per month & $46(9.0)$ & $43(8.4)$ & $89(8.7)$ & \\
\hline \multirow[t]{4}{*}{ Fat } & Once a week & $35(6.8)$ & $23(4.5)$ & $58(5.7)$ & $<0.01$ \\
\hline & Two to four times per week & $13(2.5)$ & $27(5.3)$ & $40(3.9)$ & \\
\hline & Once a day & $2(0.4)$ & $4(0.8)$ & $6(0.6)$ & \\
\hline & Twice or more per day & - & $13(2.5)$ & $13(1.3)$ & \\
\hline
\end{tabular}


Table 4. Frequency of meal, sedentary lifestyle and physical activity of adolescents among government and private secondary schools of Addis Ababa, 2012.

\begin{tabular}{|c|c|c|c|c|}
\hline \multirow{3}{*}{ Variables } & \multicolumn{3}{|l|}{ School type } & \multirow{3}{*}{ P-value } \\
\hline & Government & Private & Total & \\
\hline & $(n=512) n(\%)$ & $(n=512) n(\%)$ & $(n=1024)$ Number $(\%)$ & \\
\hline \multicolumn{5}{|l|}{ Frequency of meal per day } \\
\hline Once a day & $7(1.4)$ & 0 & $7(0.7)$ & \multirow{4}{*}{$<0.01$} \\
\hline Twice a day & $53(10.4)$ & $20(3.9)$ & $73(7.1)$ & \\
\hline Three times a day & $254(49.6)$ & $182(35.5)$ & $436(42.6)$ & \\
\hline More than three times a day & $198(38.7)$ & $310(60.5)$ & $508(49.6)$ & \\
\hline \multicolumn{5}{|l|}{ Sleeping hours per day } \\
\hline$<6 \mathrm{hrs}$ & $125(24.4)$ & $120(23.4)$ & $245(23.9)$ & \multirow{3}{*}{0.16} \\
\hline 6-9 hrs & $250(48.8)$ & $278(54.3)$ & $528(51.6)$ & \\
\hline $9 \mathrm{hrs}$ & $137(26.8)$ & $114(22.3)$ & $251(24.5)$ & \\
\hline \multicolumn{5}{|l|}{ Transport } \\
\hline Foot & $360(70.3)$ & $141(27.5)$ & $501(49.0)$ & \multirow{3}{*}{$<0.01$} \\
\hline Bicycle & $14(2.7)$ & $12(2.3)$ & $26(2.5)$ & \\
\hline Car & $138(27.0)$ & $359(70.2)$ & $497(48.5)$ & \\
\hline \multicolumn{5}{|c|}{ Time spent on TV, Video games and computer per day } \\
\hline$<30 \min$ & $107(20.9)$ & $56(0.9)$ & $163(15.9)$ & \multirow{5}{*}{$<0.01$} \\
\hline $30-60 \mathrm{~min}$ & $83(16.2)$ & $69(13.5)$ & $152(14.8)$ & \\
\hline $60-90 \mathrm{~min}$ & $66(12.9)$ & $58(11.3)$ & $124(12.1)$ & \\
\hline $90-120 \mathrm{~min}$ & $49(9.6)$ & $53(10.4)$ & $102(10)$ & \\
\hline$>2$ hours & $207(40.4)$ & $276(64.0)$ & $483(47.2)$ & \\
\hline \multicolumn{5}{|l|}{ Physical activity } \\
\hline Low & $135(26.4)$ & $219(42.8)$ & $354(34.6)$ & \multirow{3}{*}{$<0.01$} \\
\hline Moderate & $367(71.7)$ & & $660(64.5)$ & \\
\hline High & $10(2.0)$ & 293(57.2) - & $10(1.0)$ & \\
\hline
\end{tabular}

Table 5. Contextual factors of overweight/obesity among adolescents in government and private Secondary schools of Addis Ababa, 2012.

\begin{tabular}{|c|c|c|c|c|}
\hline \multirow{3}{*}{ Variables } & \multicolumn{2}{|c|}{ Overweight/obesity } & \multirow{3}{*}{ Crude OR (95\% CI) } & \multirow{3}{*}{ Adjusted OR (95\% CI) } \\
\hline & Yes & No & & \\
\hline & n(\%) & n(\%) & & \\
\hline \multicolumn{5}{|c|}{ Socio-demographic variables } \\
\hline \multicolumn{5}{|c|}{ Type of School } \\
\hline Private & $65(12.7)$ & $447(87.3)$ & $3.24(1.96-5.34)^{*}$ & $2.23(1.17-4.24)^{*}$ \\
\hline Government & $22(4.3)$ & $490(95.7)$ & 1.00 & 1.00 \\
\hline \multicolumn{5}{|l|}{ Sex } \\
\hline Female & $57(11.3)$ & $446(88.7)$ & $2.09(1.32-3.31)^{*}$ & $1.63(0.97-2.75)$ \\
\hline Male & $30(5.8)$ & 491(94.2) & 1.00 & 1.00 \\
\hline Age & & & $0.80(0.67-0.92)$ & $0.85(0.72-1.01)$ \\
\hline \multicolumn{5}{|l|}{ Family size } \\
\hline More than five & $45(9.3)$ & 437(90.7) & $1.23(0.79-1.90)$ & \\
\hline Five or less & $42(7.7)$ & $500(92.7)$ & 1.00 & \\
\hline \multicolumn{5}{|c|}{ Father's educational status } \\
\hline$>$ grade 8 & $71(10.2)$ & $622(89.8)$ & $2.25(1.29-3.93)^{*}$ & $1.43(0.72-2.85)$ \\
\hline$\leq$ grade 8 & $16(4.8)$ & $315(95.2)$ & 1.00 & 1.00 \\
\hline \multicolumn{5}{|c|}{ Mother's educational status } \\
\hline$>$ grade 8 & $57(11.7)$ & $430(88.3)$ & \multirow{2}{*}{$2.24(1.41-3.55)^{*} 1.00$} & \multirow{2}{*}{$1.10(0.60-2.00) 1.00$} \\
\hline$\leq$ grade 8 & $30(5.6)$ & $507(94.4)$ & & \\
\hline \multicolumn{5}{|c|}{ Eating Habits Number of meals } \\
\hline$\leq 3$ meals $/$ day & $55(10.7)$ & $461(89.3)$ & $1.78(1.13-2.79)^{*}$ & $1.92(1.12-3.28)^{*}$ \\
\hline$>$ than 3 meals/day & $32(6.3)$ & $476(93.7)$ & 1.00 & 1.00 \\
\hline \multicolumn{5}{|c|}{ Frequency of breakfasting } \\
\hline Not daily & $41(15.9)$ & $217(84.1)$ & $2.96(1.89-4.63)^{*}$ & $2.25(1.36-3.75)^{*}$ \\
\hline Daily & $46(6.0)$ & $720(94.0)$ & 1.00 & 1.00 \\
\hline \multicolumn{5}{|c|}{ Frequency of School recess } \\
\hline Daily & $43(9.2)$ & $422(90.8)$ & $1.19(0.77-1.85)$ & \\
\hline Not daily & $44(7.9)$ & $515(92.1)$ & 1.00 & \\
\hline Frequency of Lunch & & & & \\
\hline
\end{tabular}




\begin{tabular}{|c|c|c|c|c|}
\hline \multirow{3}{*}{ Variables } & \multicolumn{2}{|c|}{ Overweight/obesity } & \multirow{3}{*}{ Crude OR (95\% CI) } & \multirow{3}{*}{ Adjusted OR (95\% CI) } \\
\hline & \multirow{2}{*}{$\begin{array}{l}\text { Yes } \\
\text { n(\%) }\end{array}$} & \multirow{2}{*}{\begin{tabular}{l|} 
No \\
n(\%) \\
\end{tabular}} & & \\
\hline & & & & \\
\hline Daily & $67(7.8)$ & $791(92.2)$ & $0.618(0.361 .05)$ & \\
\hline Not daily & $20(12.0)$ & $146(88.0)$ & 1.00 & \\
\hline \multicolumn{5}{|c|}{ Frequency of Snack } \\
\hline Daily & $44(8.0)$ & $509(92.0)$ & $0.86(0.55-1.34)$ & \\
\hline Not daily & $43(9.1)$ & $428(90.9)$ & 1.00 & \\
\hline \multicolumn{5}{|c|}{ Frequency of Dinner } \\
\hline Not daily & $42(16.6)$ & $211(83.4)$ & $3.21(2.05-5.02)^{*}$ & $1.62(0.96-2.76)$ \\
\hline Daily & $45(5.8)$ & $726(94.2)$ & 1.00 & 1.00 \\
\hline \multicolumn{5}{|c|}{ Consumption of fast-food } \\
\hline Daily & $16(9.5)$ & $152(90.5)$ & $1.16(0.66-2.06)$ & \\
\hline Not daily & $71(8.3)$ & $785(91.7)$ & 1.00 & \\
\hline \multicolumn{5}{|c|}{ Consumption of Cereals } \\
\hline$>$ Once a day & $17(8.9)$ & 173(91.1) & $1.07(0.62-1.87)$ & \\
\hline$\leq$ Once a day & $70(8.4)$ & 764(91.6) & 1.00 & \\
\hline \multicolumn{5}{|c|}{ Consumption of Vegetables } \\
\hline$\geq$ Once a day & $25(12.3)$ & $178(87.7)$ & $1.7(1.05-2.81)^{*}$ & $1.00(0.57-1.78)$ \\
\hline$<$ Once a day & $62(7.6)$ & $759(92.4)$ & 1.00 & 1.00 \\
\hline \multicolumn{5}{|c|}{ Consumption of Fruit } \\
\hline$\geq$ Once a day & $33(13.3)$ & $215(86.7)$ & $2.05(1.29-3.25)^{*}$ & $1.22(0.71-2.09)$ \\
\hline$<$ Once a day & $54(7.0)$ & $722(93.0)$ & 1.00 & 1.00 \\
\hline \multicolumn{5}{|c|}{ Consumption of Milk \& derivatives } \\
\hline$>$ Once a day & $11(21.6)$ & $40(78.4)$ & $3.25(1.60-6.58)^{*}$ & $2.64(1.14-6.13)^{*}$ \\
\hline$\leq$ Once a day & $76(7.8)$ & $897(92.2)$ & 1.00 & 1.00 \\
\hline \multicolumn{5}{|c|}{ Consumption of Meat, Egg \& Fish } \\
\hline$>$ Once a day & $17(21.8)$ & $61(78.2)$ & $3.49(1.93-6.29)^{*}$ & $1.93(0.94-3.96)$ \\
\hline$\leq$ Once a day & $70(7.4)$ & $876(92.6)$ & 1.00 & 1.00 \\
\hline \multicolumn{5}{|c|}{ Consumption of Sweet \& Sugars } \\
\hline$>$ Once a day & $12(10.6)$ & 101(89.4) & $1.32(0.69-2.52)$ & \\
\hline$\leq$ Once a day & $75(8.2)$ & $836(91.8)$ & 1.00 & \\
\hline \multicolumn{5}{|c|}{ Consumption of Fat } \\
\hline$>$ Once a week & $3(5.1)$ & $56(94.9)$ & $0.56(0.17-1.83)$ & \\
\hline$\leq$ Once a week & $84(8.7)$ & $881(91.3)$ & 1.00 & \\
\hline \multicolumn{5}{|c|}{ Physical activity pattern } \\
\hline \multicolumn{5}{|c|}{ Time spent on TV, Computer \& Video } \\
\hline$>120 \mathrm{~min} /$ day & $52(10.8)$ & $431(89.2)$ & $1.74(1.11-2.73)^{*}$ & $1.24(0.76-2.02)$ \\
\hline$\leq 120 \mathrm{~min} /$ day & $35(6.5)$ & $506(93.5)$ & 1.00 & 1.00 \\
\hline \multicolumn{5}{|l|}{ Transport } \\
\hline Car & $57(11.5)$ & $440(88.5)$ & $2.15(1.35-3.40)^{*}$ & $1.20(0.69-2.08)$ \\
\hline Foot/Bicycle & $30(5.7)$ & 497(94.3) & 1.00 & 1.00 \\
\hline \multicolumn{5}{|l|}{ Hours of sleep } \\
\hline$>9$ hours & $16(6.4)$ & $235(93.6)$ & $0.67(0.38-1.18)$ & \\
\hline$\leq 9$ hours & $71(9.2)$ & $702(90.8)$ & 1.00 & \\
\hline \multicolumn{5}{|l|}{ Physical activity } \\
\hline Low & $37(10.5)$ & $317(89.5)$ & $1.45(0.93-2.26)$ & \\
\hline Moderate/high & $50(7.5)$ & $620(92.5)$ & 1.00 & \\
\hline
\end{tabular}

* = Statistically significant

\subsection{Contextual Factors (Determinants) of Overweight/Obesity}

Socio-demographic determinants: contextual factors of overweight/obesity are indicated in Table 5. Being a female was found to be a risk factor for overweight/obesity with $\mathrm{COR}=2.1(95 \% \mathrm{CI} ; 1.3,3.3)$. Adolescents in private schools were 3 times more likely to be overweight /obese, $\mathrm{AOR}=2.2(95 \% \quad \mathrm{CI} ; 1.2,4.2)$ compared with that of government schools. Fathers' and mothers' educational status (being above grade eight) was also a risk factor for overweight and obesity with $\mathrm{COR}=2.3(95 \% ; 1.3,3.9)$ and $\mathrm{COR}=2.2(95 \% \mathrm{CI} ; 1.4,3.5)$ respectively. Bivariate analysis using logistic regression was done for age, religion, marital status, family size, occupational status of fathers' and mothers' of the respondents; and none of them were statistically associated with overweight/obesity.

Determinants related to eating Habit: less than three meals 
per day $\mathrm{AOR}=1.9(95 \% \mathrm{CI} ; 1.1,3.3)$, lacking daily breakfast $\mathrm{AOR}=2.4(95 \% \quad \mathrm{CI} ; \quad 1.4,3.8)$, lacking daily dinner $\mathrm{COR}=3.2(95 \% \mathrm{CI} ; 2.1,5.0)$ were statistically associated to overweight/obesity. Eating vegetables, fruits, milk and milk products and meat more than once a day were also analyzed and found statistically associated to overweight/obesity with $[\mathrm{COR}=1.7 ; 1.1,2.8],[\mathrm{COR}=2.1 ; 1.3,3.3],[\mathrm{AOR}=2.7 ; 1.1$, $6.1]$ and $[\mathrm{COR}=3.5 ; 1.9,6.3]$, respectively.

Determinants related to Physical Activity: Sedentary activity (watching TV, using the computer and playing videogames) for over 120 minutes increased odds of being overweight/obese almost by two fold $[\mathrm{COR}=1.8 ; 1.1,2.7]$. Using car as a means of transport from home to school and from school to home also increased the odds of being overweight/obese by two fold $[\mathrm{COR}=2.2 ; 1.4,3.4]$. Physical activity pattern (low, moderate and high physical activity) did not show statistically significant association with overweight/obesity.

\section{Discussion}

Nutritional status of adolescents: The mean height-for-age and BMI-for-age Z- score of the study populations were $0.72 \mathrm{SD}$ and $-0.48 \mathrm{SD}$, respectively. Although the mean BAZ and HAZ of the study subjects were better compared to rural communities of Tigray, they were below the reference curve compared to the WHO 2007 reference population [3]. The reason of scoring lower mean of BAZ and HAZ than the WHO growth reference might be due to their poor dieting habit which is not according to the recommended dietary intake. The prevalence of being under nutrition (stunting and underweight) among participants was $13.4 \%$ which is higher than that of adolescents in Palestine (4.8\%) [17] and 8.7\% reported from Jamaica [18] while it is much lower than that of adolescent girls from rural communities of Tigray (58.3\%) [3], and Myanmar (32\%) [19]. The prevalence of under nutrition in adolescents is terribly high compared to $4.8 \%$ reported in Palestine since under nutrition may affect the maturation and later physical work capacity.

\subsection{Contextual Factors of Overweight/Obesity Related to Socio-Demographic Variables}

The magnitude of overweight/obesity was $8.5 \%(12.7 \%$ for private and $4.3 \%$ for government school students) which is comparable with that of Indian adolescents which ranged from (5.9-17.8\%) [7] and Addis Ababa(8.5\%) [14]. A similar study in Islamabad (14.1\%) showed that overweight/obesity was higher compared to our study subjects [1]. Another study conducted in India showed a considerably lower percentage of obesity (2.2\%) among adolescents [20]. Prevalence of overweight/obesity indicates remarkable difference between government and private schools in which overweight/obesity among private school students is comparable to studies from developed countries. One possible explanation for the higher percentage of overweight/obesity among private school might be due to their families' higher socioeconomic status in that it could have allowed them to adopt unhealthy nutritional habits including consumption of fast food, sweets and more animal products.

The prevalence of overweight/obesity in this study was higher among girls $(11.3 \%)$ than boys $(5.7 \%) \mathrm{OR}=2.1(955$ CI; 1.3, 3.3). This finding contradicts the findings of Nigeria [21], Brazil [6], Canada [22] and Italy [23] which generally showed a higher prevalence of overweight/obesity among boys than girls. This high prevalence of overweight/obesity among girls is worrisome since overweight/obesity persists to adulthood and has an impact on psychosocial wellbeing of adolescents related to negative body image, low self esteem, and anxiety or depression, especially in girls [24,25]. Although fathers' and mothers' educational status above grade eight increases the odds of getting overweight/obesity by 2.4 times than family's educational status less than or equal to grade eight, this association was lost after controlling for the possible confounders.

\subsection{Contextual Factors (Determinants) of Overweight/Obesity Related to Dietary Habit}

In the crude analysis, the act of consuming fruits and green vegetables more than or equal to once per day in our study increased the odds of getting overweight/obesity with $\mathrm{OR}=$ $1.7 \& 2.1$ respectively. The result disagrees with the findings from Canada [22], Brazil [6] and Spain [26] showing that no fruit and greens consumption was associated with overweight and obesity. It also contradicts with the epidemiological studies that suggest consumption of a major quantity of fruits and vegetables is associated with a reduction of risk for cancer and is beneficial in cardiovascular diseases, diabetes, obesity and cerebral vascular accidents [27]. The reversed effect observed might be adolescents from wealthy families in the study population might tend to consume fruit and vegetables as a supplement in addition to the regular meal but not substitute the regular meal.

Eating animal product foods (milk, yogurt and cheese) more than once a day showed a significant association with overweight/obesity $(\mathrm{AOR}=2.6,95 \% \mathrm{CI} ; 1.1,6.1)$. This result is consistent with a study conducted among children and adolescents in elementary schools of Addis Ababa [14]. The fact behind this association might be animal product yields high energy that may lead to excess weight gain.

Lacking daily breakfast and having meals less than three times a day proved to be a risk factor for overweight/obesity $(\mathrm{AOR}=2.3,95 \% \mathrm{CI} ; 1.4,3.8$ and $1.9,95 \% \mathrm{CI} ; 1.12,3.28$, respectively). This agrees with studies from Brazil [6] where the habit of no breakfasting daily was a risk factor for overweight and obesity; another study conducted elsewhere also showed that overweight children had breakfast less frequently than the thin ones [28]; and breakfasting regularly in the morning is associated to lower levels of cholesterol and to low weight [29, 30]. This study also agrees with another study from US in which frequent restrictive dieting contribute to slowed metabolic rate, binge eating and weight gain [31]. This might be true in that adolescents who are overweight/obese in this study were trying to control their weight by decreasing the number of meal omitting their 
breakfast.

AOR: Adjusted for school type, sex, age, family educational status, meal frequency, frequency of breakfast and dinner, consumption of vegetables, consumption of milk products, consumption of animal products, time spent on TV, computer and video, means of transport and physical activity.

Determinants of overweight/obesity related to physical activity: Boys were more physically active than girls (Pvalue $<0.01)$. This disparity of physical activity patterns between the sexes agrees with the reports from Palestine and US $[15,17]$. The same trend was noticed for physical activity as boys are more physically active than girls. The study showed no association between the practice of physical activity and overweight/obesity. However, the tool used to evaluate this kind of activity was not specific for adolescents although it was validated in a population that included individuals aged from 12 years old [6].

The results of this assessment showed that the time spent watching TV, playing videogames and computers $(>120$ minutes/day) presented a significant association with overweight/obesity in crude analysis, losing significance in the multivariate analysis. Other studies performed in Brazil [6] and England [15] agrees with this result. Some authors, however, do not consider the habit of watching TV, playing videogame or using the computer as markers for inactivity in the association between sedentarism and overweight [32]. The proportion of secondary school adolescents walking to school $(49 \%)$, being driven to school $(48.5 \%)$ and cycling to school $(2.5 \%)$ was comparable to results from developed countries like England in which the proportion of walking, driven and cycling was $56 \%, 42 \%$ and $2 \%$ respectively [15]. Changes in patterns of physical activity and the adoption of more sedentary lifestyles are likely to be important factors behind overweight/obesity.

Conclusions and recommendations: Findings of the present study indicated that both under and over nutrition are important problems of adolescents in government and private secondary schools. Adolescents in private schools, sedentary lifestyle, lacking regular consumption of breakfast, eating meal less than 3 times per day, and consuming milk, yogurt and cheese more than once a day significantly influenced the overweight/obesity of adolescents. Hence, it is recommended that there should be targeted educational intervention programmes on sedentary activities, Health and nutrition. Furthermore, youth centers and schools should encourage opportunities for low/no cost physical activities.

Limitation of the study: Since the study was cross sectional study it was difficult to establish a direct cause and effect relationship between risk factors and outcome variable. During interview there might be social desirability bias by participants. The absence of qualitative methods is another limitation of this study.

Acknowledgment: Our deepest gratitude goes to Addis Ababa University for funding this research. Our sincere thanks also goes to the schools' principals and study subjects for participating in the study.

Abbreviations: AOR -Adjusted Odds Ratio; BMI-Body
Mass Index; BAZ-BMI-for-Age Z-score, CI-Confidence Interval; COR-Crude Odds Ratio;FAO- Food and Agriculture Organization; FFQ-Food Frequency Questionnaire; HAZHeight-for-Age Z-score; IPAQ-International Physical Activity Questionnaire; OR-Odds Ratio; USA-United States of America; WHO-World Health Organization.

\section{References}

[1] Ahmad H, Liaqat P, Paracha PI, Qayyum A and Uppal A. Assessment of Nutritional Status of Adolescents versus Eating Practices in Islamabad City. Pakistan Journal of Nutrition, vol. 8(8), pp.1304-08, 2009.

[2] Meseret Y. Anthropometric Assessment of Adolescent Malnutrition in Elementary and Secondary Schools of Ambo Town,West Shewa Zone, Oromia Region, Ethiopia. MPH thesis, Addis Ababa University, 2008.

[3] Afework M, Fitsum H, Barbara S, Gideon K, Vincent L, Zenebe A et al. Nutritional Status of Adolescent Girls from Rural Communities of Tigray, Northern Ethiopia. EthiopJHealth Dev, vol. 23(1), pp.5-11, 2009.

[4] Anyika J, Uwaegbute, Olojede A and Nwamarah J. Nutrient Intakes of Adolescent Girls in Secondary Schools and Universities in Abia State of Nigeria. Pakistan Journal of Nutrition, vol. 8(10), pp.1596-602, 2009.

[5] Kalhan M, Vashisht B, kumar V and Sharma S. Nutritional Status of adolescent girls of rural Haryana.The Internet Journal of Epidemiology, vol. 8(1), pp.67-75, 2010.

[6] Carvalho IH, Francescantonio M, Neutzling M and Taddei A. Risk factors for overweight and obesity in adolescents of a Brazilian university: a case-control study. Nutr Hosp, vol. 24, pp.17-24, 2009.

[7] VanderKloet M. Dual Burden of Malnutrition in Andhra Pradesh, India: Identification of Independent Predictors for Underweight and Overweight in Adolescents with Overweight Mothers. Research for Develoipment record, September 2008.

[8] WHO. Developing countries face double burdened of disease, bulletin of the WHO, 82(7) Genebra, July 2004.

[9] David H, Mc Carth, Sandra M and Tim J. Central overweight and obesity in British youth aged 11-16 years: cross sectional surveys of waist circumference. BMJ, vol. 326(7390), pp.624, 2004.

[10] Stice E, Presnell K and Shaw H. Psychological and Behavioral Risk Factors for Obesity Onset in Adolescent Girls: A Prospective Study. Journal of Consulting and Clinical Psychology, vol. 73(2), pp.195-202, 2005.

[11] Stray-Pedersen M, Helsing R, Gibbons L, Cormick G, Holmen T, Vik T et al. Weight status and hypertension among adolescent girls in Argentina and Norway: Data from the ENNyS and HUNT studies. BMC Public Health, vol. 9, pp. 398, Oct 2009.

[12] Deshmukh P, Gupta S, Bharambe M, Dongre M, Kaur S and Garg B. Nutritional Status of Adolescents in Rural Wardha Indian. J Pediatrics,vol.73 (2), pp.139-41, 2006.

[13] WHO anthroplus manual. Department of Nutrition for Health and Development. Geneva, Switzerland, 2009. 
[14] Zeleke A. Prevalence of childhood and adolescent overweight and obesity among elementary school students in Addis Ababa duble burden of malnutrition in Ethiopia. .Addis Ababa University Libraries Electronic Thesis and Dissertations, November 2007.

[15] Reilly JJ et al. Childhood obesity in United Kingdom. British Medical Journal, September 2003.

[16] Weight control Information Network, WIN.statistics related to overweight and obesity. NIDDK, 2010.

[17] Jildeh C, Papandreou C, Mourad TA, Hatzis C, Kafatos A, Qasrawi $R$ et al. Assessing the Nutritional Status of Palestinian Adolescents from East Jerusalem: School-based Study. Journal of Tropical Pediatrics, 2002-03.

[18] Jackson M, Samms-Vaughan M and Ashley D. Nutritional status of 11-12 years-old Jamaican children: coexistence of under and over-nutrition in early adolescence. Public Health Nutr, vol. 5(2), pp.281-288, 2002.

[19] WHO Regional Office for South-East Asia New Delhi. Improvement of Nutritional Status of Adolescents. Report of the Regional Meeting Chandigarh, India, 17-19 September 2002.

[20] Wang Y. Cross-sectional comparison of childhood obesity: The epidemic and the relationship between obesity and socioeconomic status International journal of Epidemiology, vol.30, pp.1129-36, 2001.

[21] Ejike EC, Ugwu EC and Ezeanyika L. Physical Growth and Nutritional Status of a Cohort of Semi-Urban Nigerian Adolescents. Pakistan Journal of Nutrition, vol. 9(4), pp.392397, 2010.

[22] Janssen I, Katzmarzyk PT, Boyce WF, King MA and Pickett W. Overweight and obesity in Canadian adolescents and their associationswith dietary habits and physical activity patterns. J Adolesc Health, vol. 35, pp.360-7, 2004.

[23] Celi F, Bini V, De-Giorgi G et al. Epidemiology of overweight and obesity among school children and adolescents in three provinces of central Italy: study of potential influencing variables. Eur. J. Clin Nutr, vol. 57, pp.1045-51, 2003.

[24] Dietz WH. Critical periods in childhood for the development of obesity. Am.J. Clin. Nutr, vol.59, pp.955-9, 1994.

[25] National Center for Health Statistics. Update: Prevalence of overweight among children, adolescents and adults, United States. Morb Mortality Weekly Report, vol. 46(9), pp. 199201, 1997.

[26] Izaga M, Rocandio A, Ansotegui L, Apalauza P, Salces I and Ochoa E. Diet quality, overweight and obesity in university students. Nutr Hosp, vol. 21, pp.673-9, 2006.

[27] Jiménez-Cruz A, Bacardí-Gascón $M$ and Jones E. Consumption of fruits, vegetables, soft drinks, and high-fatcontaining snacks among Mexican children on the MexicoU.S. border. Arch Med Res, vol.33, pp.74-80, 2002.

[28] Kumar B, Holmboe-Ottesen G, Lien N and Wandel M. Ethnic differences in body mass index and associated factors of adolescents from minorities in Oslo, Norway: a crosssectional study. Public Health Nutr, vol.7, pp.999-1008, 2004.

[29] Ruxton CHS and Kirk TR. Breakfast: a review of associations with measures of dietary intake, physiology and biochemistry. Br J Nutr, vol.78, pp.199-213, 1997.

[30] Sjoberg A, Hallberg L, Hoglund D and Hulthen L. Meal pattern, food choice, nutrient intake and lifestyle factors in the Goteborg Adolescence Study. Eur J Clin Nutr, vol.57, pp.1569-78, 2003.

[31] National Task Force on the Prevention and Treatment of Obesity. Overweight, obesity, and health risk. Arch Intern Med, vol. 160(7), pp.898-904, 2000.

[32] Marshall SJ, Biddle SJ, Gorely T, Cameron N and Murdey I. Relationships between media use, body fatness and physical activity in children and youth: a meta-analysis. Int J Obes Relat Metab Disord, vol.28, pp.1238-46, 2004. 